\title{
Analysing and Forecasting Electricity Demand and Price Using Deep Learning Model during the COVID-19 Pandemic
} \author{
Israt Fatema ${ }^{1[0000-0002-2248-2472]}, \underset{0003-0845-6718]}{1[1111-2222-3333-4444]}$ and Gengfa Fang ${ }^{1[0000-}$ \\ ${ }^{1}$ University of Technology Sydney, NSW 2007 \\ israt.fatema@student.uts.edu.au
}

\begin{abstract}
The smart city integrating the smart grid as an integral part of it to guarantee the ever-increasing electricity demand. After the recent outbreak of the COVID-19 pandemic, the socioeconomic severances affecting total levels of electricity demand, price, and usage trends. These unanticipated changes introducing new uncertainties in short-term demand forecasting since its result depends on the recent usage as an input variable. Addressing this challenging situation, this paper proposes an electricity demand and price forecast model based on the LSTM Deep Learning method considering the recent demand trends. Real electricity market data from the Australian Energy Market Operator (AEMO) is used to validate the effectiveness of the proposed model and elaborated with two scenarios to get a wider context of the pandemic impact. Exploratory data analyses results show hourly electricity demand and price reductions throughout the pandemic weeks, especially during peak hours of 8 am- 12 noon and $6 \mathrm{pm}-10$ pm. Electricity demand and price has been dropped by $3 \%$ and $42 \%$ respectively on average. However, overall usage patterns have not changed significantly compared to the same period last year. The predictive accuracy of the proposed model is quite effective with an acceptably smaller error despite trend change phenomena triggered by the pandemic. The model performance is comprehensively compared with a few conventional forecast methods, Support Vector Machine (SVM) and Regression Tree (RT), and as a result, the performance indices RMSE and MAE have been improved using the proposed LSTM model.
\end{abstract}

Keywords: COVID-19, pandemic, electricity demand and price forecast, LSTM, smart grid, smart city.

\section{$1 \quad$ Introduction}

Smart City can efficiently address the challenges of a growing population to manage their essential activities, such as energy, transport, health, and homes. A reliable and sustainable Smart Grid (SG) is essential for affordable electricity to smart city's consumers. However, the pandemic of COVID-19 affects almost all aspects of the community and eventually the concept of a smart grid. Due to the present circumstances of COVID-19, mass people are working from home, and the forced closure of industries 
and other commercial activities significantly slacked down daily activities in comparison with the non-pandemic ones. The socioeconomic severances naturally affecting on total levels of electricity consumption, demand, price, and usage trends worldwide.

This changed working condition eventually reflected in electricity grid planning, demand scheduling, renewable source integrating, and spot pricing. Electricity demand and price forecasting have important roles in the economy, which is frequently used in business planning, policymaking, and market setting. Smart grids are depended on a forecasting model that is mostly designed and validated on historical data. However, there are no historical time-series smart grid data presents comparable to the COVID19 pandemic period. Consequently, the short-term forecasting algorithm's performance is affected by the aforementioned uncertainty. Therefore it is imperative to improve the forecasting accuracy in terms of the possible error reduction.

Traditional forecasting methods, such as moving average (MA) and trend analysis, get complicated and limited if used in large time-series data set [1]. These methods are challenging to accurately measured and represented with detail dynamic operations occurred because of the recent trend shift. Researchers have applied deep-learning methods, such as Artificial Neural Network (ANN), to improve the models' prediction accuracy by reducing errors and modelling complex patterns. In the context of such uncertain times of pandemics, this paper aims to contribute and address the problems of implementing accurate and reliable demand and price forecast algorithm. First, an initial exploratory analysis of electricity demand and price time -series data is performed to compare the diurnal variation during the pandemic and non-pandemic periods. Statistical analysis is applied to evaluate the inconsistency and uncertainty of the forecasting problem. Secondly, a single comprehensive model of the LSTM based sequenceto-sequence network is proposed to forecast electricity demand and price. This model can effectively learn variable temporal correlation in the input sequence, which is commonly used for language translation [2]. Similar temporal correlations are also present in the electricity demand and price pattern.

The arrangement of this paper is as follows: related work is given in Sect. 2. The theoretical background of this work is discussed in Sect.3. Section 4 describes the dataset used for this study. An empirical analysis of data is in Sect.5. The modeling results and discussions are presented in Sect. 6. Section 7 concludes the article.

\section{Related Work}

Deep learning has been increasingly involved in the forecasting methods [3] and is being applied effectively in various time series issues, such as language modelling [4], speech recognition [5], stock market prediction [6], and flood forecasting [7]. In [8], the researchers used a recurrent neural network (RNN) as an important approach for time-series forecasting. However, RNN shows inadequacy in learning long term dependencies and relies on fixed-term to learn time-series sequence computation [9]. LSTMs, are a special type of RNN, could learn long-term dependencies by remembering and collecting information of time-series [10]. Few other approaches were also implemented to enhance electricity demand or price forecasting performance, such as 
feature selection and genetic algorithm to optimize a LSTM model [11], support vector regression (SVR), stacked auto-encoders (SAEs) with the extreme learning machine (ELM) [12], and stacked de-noising auto-encoders with SVR [13]. It is observable that the ANN-RNN based models are very common for related fields of electricity.

The aforementioned methods could give good results, yet its algorithms are complicated and difficult. This paper, therefore, proposes a single comprehensive model of the LSTM based sequence-to-sequence network to forecast electricity demand and price. Though such a model has recently been used in energy and weather forecasts, its application has not been used widely in terms of electricity demand and price forecast. In [14], LSTM was compared to the sequence-to-sequence network and in [15], sequence-to-sequence RNN was compared to standard RNN. In both studies, the sequence-to-sequence network provided better results than other models. A sequence-tosequence RNN was developed with an attention mechanism for the electric load forecast in recent research [15], and a similar sample generation method was designed. In [16], A LSTM-based short-term load forecast model with two mechanisms is built. Their approach is similar to the language translation model in [2], where one LSTM is used to encode the input sequence into a fixed vector, and then need separate LSTM to decode the vector to a sequence of outputs with an attention mechanism to learn weight. The attention mechanism learns to weight the input features variable conditioned on the previous input(s) rather than fixed weighting features [2]. Alternatively, this research uses a simplified approach using a single LSTM that encodes and decodes both on the basis of the given inputs. This allows the LSTM to share weights between encoding and decoding. Therefore, no complicated attention mechanism is required for this straightforward sequence-to-sequence model.

\section{Theoretical Background}

\subsection{Long Short Term Memory (LSTM) Network}

The LSTM's key objective is to prevent the issue of vanishing gradient which occurs while training of backpropagation neural network (NN), and thus limiting the model's ability to learn long-term temporal correlations [10]. All RNN follow the structure of a chain of recurring modules of NN. For regular RNNs, this recurring module will have a very simple structure, like a single tanh layer. The structure of LSTM includes additional three main gate structures: forget gate $\left(f_{t}\right)$, input gate $\left(i_{t}\right)$, and output gate $\left(o_{t}\right)$. Based on the LSTM unit defined in [17], for an input $x_{t}$ at time step t, the LSTM calculate a hidden state $h_{t}$ and memory cell state $C_{t}$ to encode all the observed state by the cell till time $t$. The LSTM network computes a mapping from an input sequence $X=$ $\left(x_{1}, x_{2}, \ldots, x_{n}\right)$ to an output sequence $\mathrm{Y}=\left(y_{1}, y_{2} \ldots, y_{m}\right)$. The LSTM cell computation at time $t$, for an input $x_{t}$ :

$$
\begin{gathered}
f_{t}=\delta\left(W_{f} x_{t}+U_{f} h_{t-1}+b_{f}\right) \\
i_{t}=\delta\left(W_{i} x_{t}+U_{i} h_{t-1}+b_{i}\right)
\end{gathered}
$$




$$
\begin{gathered}
g_{t}=\tanh \left(W_{g} x_{t}+U_{g} h_{t-1}+b_{g}\right) \\
C_{t}=i_{t} * g_{t}+f_{t} * C_{t-1} \\
o_{t}=\delta\left(W_{o} x_{t}+U_{o} h_{t-1}+b_{o}\right) \\
h_{t}=o_{t} * \tanh C_{t}
\end{gathered}
$$

where $\delta$ and tanh are the activation function, $W$ and $U$ are the weight of forget gate, and $b$ is the bias vector, $C_{t-1}$ and $C_{t}$ are the cell states at time $t-1$ and $t$.

\subsection{Sequence-to-Sequence Network}

To forecast the values of future time steps of a sequence, a sequence-to-sequence regression LSTM network can be trained. A typical sequence-to-sequence model consists of two phases, an encoder, and a decoder. It can take input sequence $\mathrm{X}$ (encoder) of variable length and change that in a fixed-length vector, which is then used as the input sequences for the next time step [2]. Hereby an output sequence $\mathrm{Y}$ (decoder) of $\mathrm{n}$ length is generated. In this case, at each time step of the input sequence, the LSTM network learns to forecast the value of the next $\mathrm{n}$ time steps. Therefore, during encoding, with input sequence X, the LSTM computes a sequence of hidden states $\left(h_{1}, h_{2}, \ldots, h_{n}\right)$. During decoding it defines a distribution over the output sequence $\mathrm{Y}$ given the input sequence $\mathrm{X}$ as $\mathrm{p}(\mathrm{Y} \mid \mathrm{X})$ is:

$$
p\left(y_{1}, \ldots, y_{m} \mid x_{1}, \ldots, x_{n}\right)=\prod_{t=1}^{m} p\left(y_{t} \mid v, y_{1}, \ldots, y_{t-1}\right)
$$

where $v$ is fixed dimensional vector representation of the $X$ given by the last hidden state based on the recursion of the LSTM Eqs. (1) - (6), and the distribution of $p\left(y_{t} \mid v, y_{1}, \ldots, y_{t-1}\right)$ is given by a softmax function (Eq. 8) to create a probability vector that helps determine the final output.

$$
p(y)=\operatorname{softmax}\left(w^{t} h_{t}\right)
$$

where the softmax activation function calculates the probability of each recurrent weight using the state hidden $\left(h_{t)}\right)$ at current time step with each weight $\left(w^{t}\right)$.

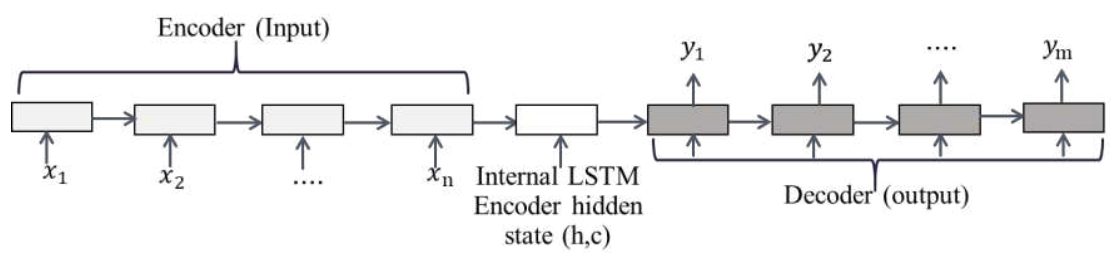

Fig. 1. The structure of Sequence-to-Sequence Network, $(h, c)$ represents intermediate vector

The proposed method, shown in Fig. 1, relies on a single LSTM for both the encoding and decoding phases. Thus parameter is shared within the encoding and decoding phases. The proposed model uses two LSTMs layers, the hidden state $\left(h_{t}\right)$ from the first LSTM layer is given as the input $\left(x_{t}\right)$ to the second LSTM. The first LSTM layer is used to create the input sequence for time series data and the second layer is used to 
create the prediction by the next output sequence. Forecasting model performance is measured by Root Mean Squared Error (RMSE), Mean Absolute Error (MAE), and correlation coefficient value $\left(\mathrm{R}^{2}\right)$. A lower RMSE and MAE indicates better result, which measures the difference between the actual and forecast values. The $\mathrm{R}^{2}$ value is between 0 and 1 ( 0 means no correlation and 1 means no error), determines the correlation between actual and predicted values. Figure 2 shows the step-by-step flowchart of this study that includes two steps: an analysis module and a forecasting module.

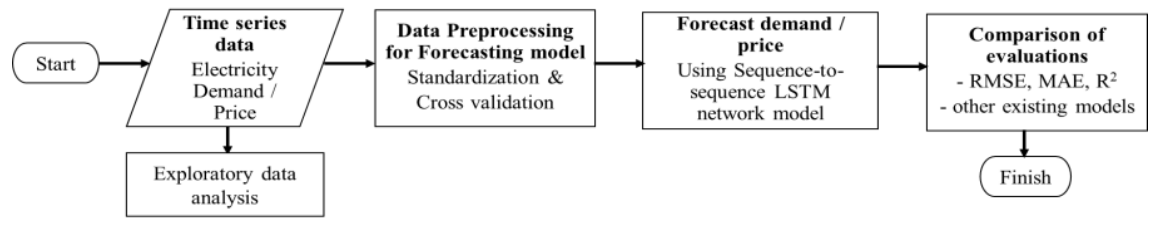

Fig. 2. Flowchart of the proposed forecasting method

\subsection{Forecasting Model Description}

The proposed model used Dropout as a regularization methodology for fully connected neural network layers to avoid over-fitting, and improving accuracy on testing data [18]. All elements of an output layer are stored with probability $p$, alternatively set to 0 with probability $(l-p)$. Eq. (12) shows in this case drop unit or not [18].

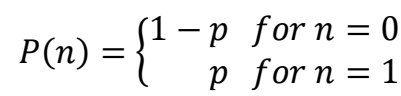

The simulation result's precision is improved by adjusting and setting the model's appropriate variables to produce the desired output. The model parameter was set in a total of 36 settings to achieve best result. The initial learning rate of the experiment was set to 0.005 , the number of hidden units was set to $200 / 100 / 50$, set the number of iterations to 300/250/200, Dropout $(\mathrm{p}=0.5)$ and Adam (Adaptive Moment Estimation) optimizer algorithm is used for optimization to update network weights.

\section{$4 \quad$ Dataset and Data Preprocessing}

It is not immensely challenging to train a forecasting algorithm using historical data. However, social trends changed quite quickly due to the COVID-19 pandemic effect, which may cause the models built using historical data ineffective and incorrect. Since there are only months of data available for model training and testing processes and small amounts of data generally decrease model accuracy. To address this issue, AEMOs' open dataset is used, which contains accumulated daily electricity demand (30 min MW) and price (30 $\mathrm{min} / \mathrm{MWh}$ ) sampling rate [19]. This study used the range of data for the forecasting model is from January 2019 to August 2020. To analyse the impact on electricity demand and price profiles during COVID-19 and to evaluate the forecasting model, this study focuses on data from two states in Australia: New South Wales (NSW) and Victoria (VIC) as two different scenarios. The reasons for selecting 
these states are: (1) NSW is the highest populated state and the restriction set to ease due to reduced infection rate, (2) VIC has the highest number of COVID 19 cases compared to other states and is experiencing a second wave, not currently being seen in other states. Consequently, VIC is under strict restrictions.

To enhance model efficiency, the cross-validation technique is used for the assessment of the forecasting model [20]. Cross-validation stages include the splitting of the data set into training, and test data for unbiased performance comparison [20]. The data split of this research is $90 \%$ training and tests on the remaining $10 \%$. The original data is pre-processed by standardization to improve the model prediction accuracy and to eliminate the training from deviating [21]. Standardize the data to have zero mean and unit variance to make it more effective:

$$
X_{\text {stand }}=\frac{X-\mu}{\sigma}
$$

Here, the standardized variable is $X_{\text {stand }}$ which is equal to the original variable (x), minus its mean $(\mu)$, divided by its standard deviation $(\sigma)$.

\section{$5 \quad$ Impact on Electricity Demand and Price during COVID-19}

The initial exploratory analysis of electricity demand and price data can be useful to better understand the dynamic changes and identify trends and patterns to the energy sector due to COVID-19. Due to the influence of several factors, such as temperature, day of the week, and variation of renewable sources, it is not easy and straightforward to determine the actual impact of the pandemic on the electricity demand and price.

In Australia, the nationwide restrictions, which started in mid-March to control the spread of COVID-19, also resulted in decreased demand for electricity and price. Figure 3 (a-d) follows four years (2017-2020) data of two seasonal patterns, autumn (MarchMay) and winter (June-August) in NSW and VIC to show a comparison of change. As shown in Fig. 3, the NSW COVID-19 related demand decrease was highest compare to other year's similar period. Regardless, demand reductions were declining from the end of May due to the winter season and easing restriction. However, VIC's COVID-19 demand reduction was steady. For both scenarios, residential demand increased due to shut-down and working from home setup, and commercial demand was reduced due to limited business activities. Cold temperatures have resulted in an average increased in residential demand in both states (Fig. 4) since it is more related to weather than other industries. Figure 3 shows a drastic price reduction in both states during the COVID19 period compare to the previous year's similar time. During the pandemic, both states recorded its lowest average price since 2016 [19]. The pandemic has contributed to a major price drop in the international and local markets for crude oil, gas, and thermal coal [19]. This is a key factor of reduced spot price along with other factors including reduced demand and increased amount of renewable generation.

Figure 4 (a-b) shows the changing pattern of daily average electricity demand, price, temperature, and renewable and non-renewable contribution to demand in NSW and VIC, during the pandemic period from 1st March to 31st August 2020 (data adopted from [22]). The figures suggest that it is difficult to capture the unexpected shifts in 
social behavior and pattern of work during the pandemic period since electricity generation (renewable and non-renewable), demand, price, and temperature change are not linear and not always following any pattern. Generally, the weather and different seasons influence the demand and price of electricity. Throughout the pandemic period, electricity demand shows a predictable trend and rises at a consistent rate to keep state with the temperature, unlike the electricity price that changes very randomly with a few sudden price peaks and intermittently following any pattern. Besides, increased renewable sources that include increased rainfall, wind, and solar production, have contributed to lower prices for both of the scenarios.

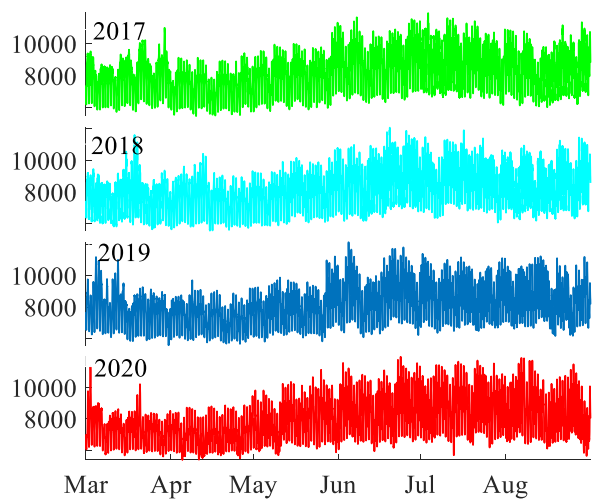

(a) NSW Demand (MW)

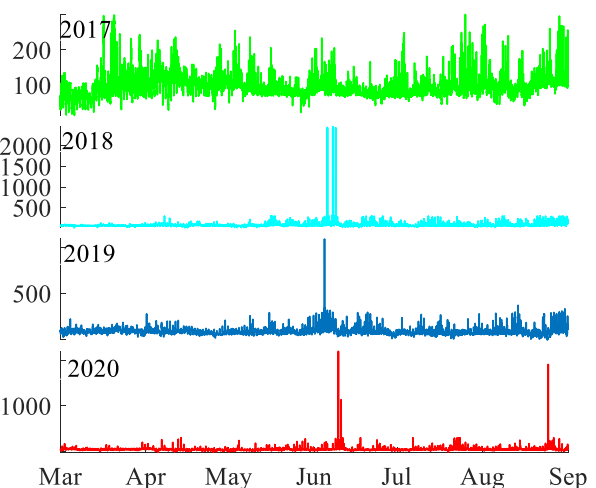

(b) NSW Price (MWh)

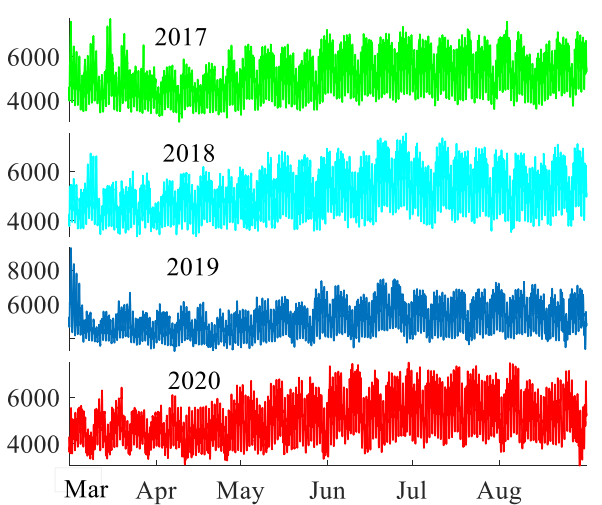

(c) VIC Demand (MW)

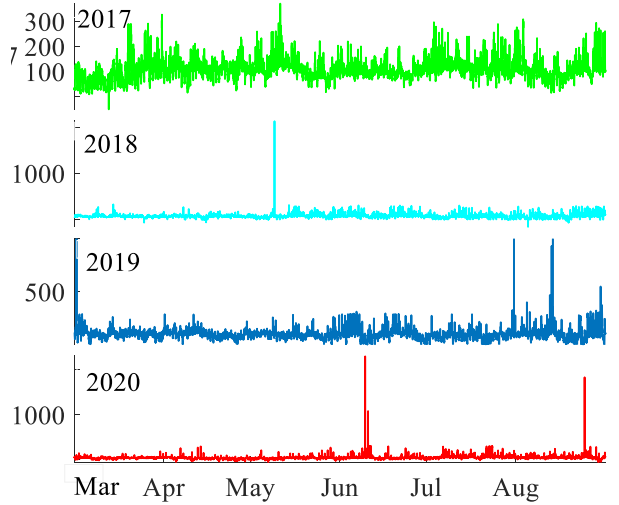

(d) VIC Price (MWh)

Fig. 3. Comparison of changing demand and price patterns for both scenarios from March-August in 2017-2020.

To analyse the daily real usage scenario during the pandemic period, demand/price data was grouped into weekdays and weekends, with the calculation of the average weekly time of use and compared to the same period of 2019. Hourly electricity demand and price show a clear reduction during the pandemic in NSW and VIC, especially during peak hours of 8 am- 12 noon and $6 \mathrm{pm}-10 \mathrm{pm}$. During the non-pandemic time, electricity price on weekdays is much higher than the weekend price, especially around 
peak hours in both scenarios. Compared with the price curve during the pandemic period, weekday and weekend are following a similar trend and overall price decreased in both peak and off-peak hours (Fig. 5).

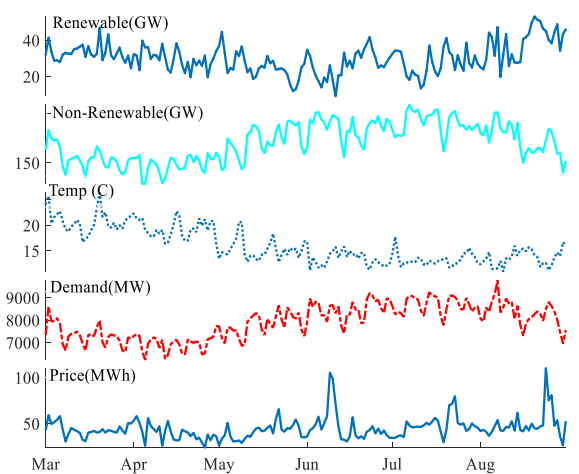

(a) NSW

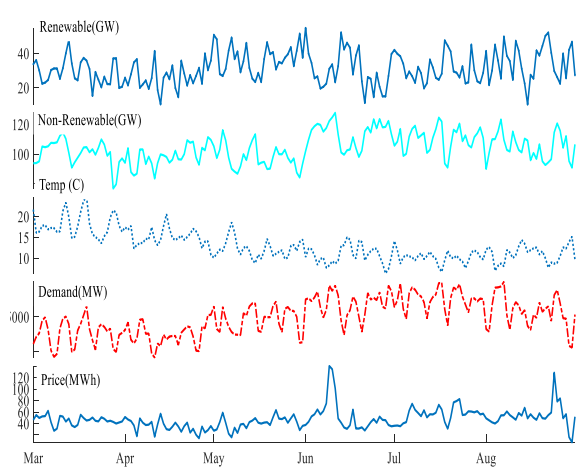

(b) VIC

Fig. 4. The changing pattern of daily average electricity demand, price, temperature, and renewable and non-renewable contribution in (a) NSW and (b) VIC during the COVID-19 period.

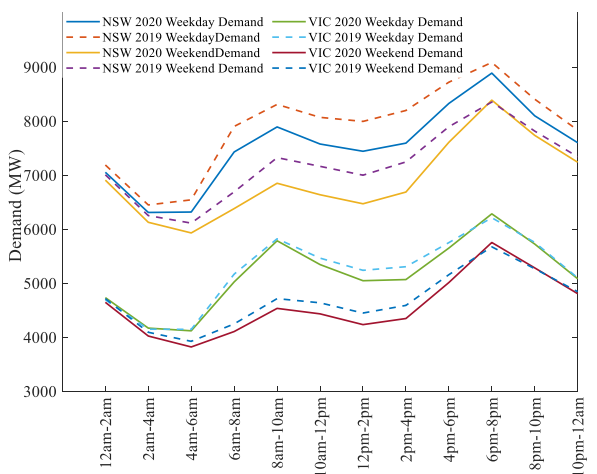

(a) Demand

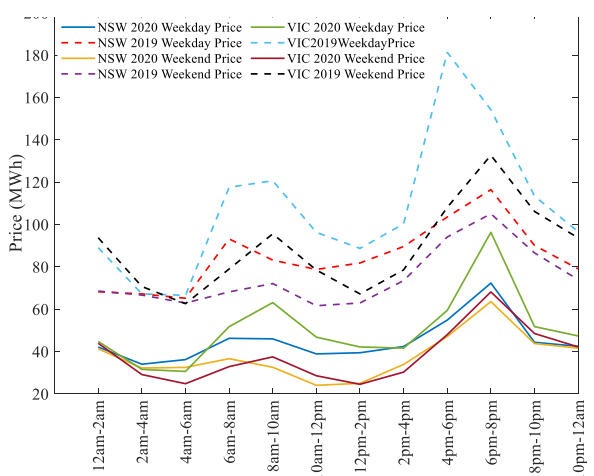

(b) Price

Fig. 5. Weekly electricity time of use during COVID-19 and the same period of 2019 in (a) NSW and VIC demand (b) NSW and VIC Price

Notably, overall demand and price patterns have not changed significantly compared to the same period last year (Fig. 5). This indicates people working from home are continuing regular activities and consuming electricity that remained usual morning and evening peak time trends during the COVID-19 pandemic. Statistical analysis is also performed to compare the changes. A Variation Index (VI) is defined which presents the average reduction of demand/price to show the diurnal variation during the COVID-19 period (2020) compared to a benchmark period (2019) as follows:

$$
V I_{i t} \frac{\sum_{i=1}^{n}\left(D_{c t}-D_{o t}\right) \times 100}{n \bar{D}}
$$

where $V I_{i t}$ is the index value of $i$ for time $t, D_{c t}$ is the current demand/price for time $t$ 
(weekday and weekend), $D_{o t}$ is the demand/price for the same time of a previous benchmark period, $n$ is the number of recorded demand/price, and $\bar{D}$ is the average demand/price during the previous benchmark period.

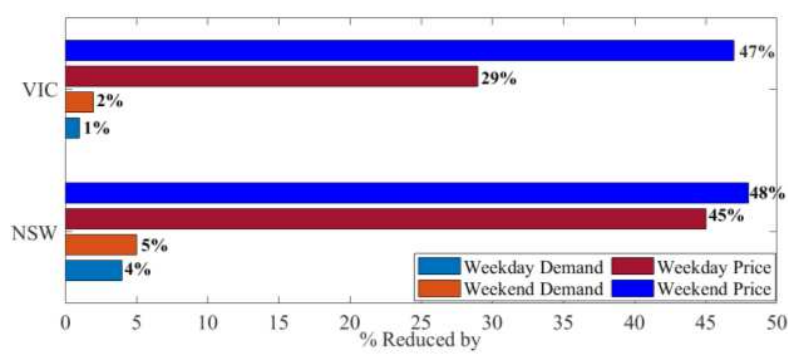

Fig. 6. Variation Index (VI) between pandemic and non-pandemic period for NSW and VIC

Figure 6 shows the VI for both of the states and the results show that NSW weekday electricity demand and price were decreased by $4 \%$ and $45 \%$ respectively on average, and on weekends it was $5 \%$ and $48 \%$ respectively during the strict restriction period (March-May). Compare to NSW, VICs' demand reduction was lower on weekdays (average $1 \%$ ), due to cooler weather. The weekend price was significantly dropped by $47 \%$ on average, whereas weekday prices reduced by only $29 \%$ on average.

\section{Forecasting Model Results And Discussion}

In this section, the proposed model forecasts the aforementioned two different scenarios (NSW and VIC) to get a wider context of the COVID-19 pandemic impact on electricity demand and price. The model has been trained and tested 9 times on independent data with different forecasting granularities (section 3.3). The simulation results explain the performance of the proposed model in addition to the RMSE and MAE errors. Table 1 summarizes the best prediction results from 36 test results of two different scenarios.

Figure 7 shows that the forecasted and the observed values mostly conform to each other. The blue line in each figure represents the observed values and the red line implies the forecasted values. The forecast values quite closely match with the observed values of electricity demand data. However, compared to the demand forecast, spot pricing is not as accurate as expected in Fig. 7 (b) and (d). Figure 8 displays the $R^{2}$ values for electricity demand and price for both scenarios. Whereas, the $R^{2}$ of the electricity price for NSW and VIC are .78 and .88 respectively, between actual and predicted values. This indicates a moderate correlation and similarity with sect. 5 analysis that price VI was high and indistinct compare to demand during the pandemic period.

Table 1. Comparison of electricity demand and price forecasting errors and test result (summary).

\begin{tabular}{rcrrrrrrrr}
\hline & \multirow{2}{*}{ RMSE } & \multirow{2}{*}{ MAE } & \multicolumn{2}{c}{ Observed } & \multicolumn{2}{c}{ Forecasted } & \multicolumn{2}{c}{ Relative error } \\
\cline { 5 - 10 } & & & High & Low & high & Low & High & Low \\
\hline \multirow{2}{*}{ NSW } & Demand & 58.21 & 40.51 & 11908.24 & 5630.73 & 11890.48 & 5567.92 & 0.17 & 0.62 \\
& Price & 29.67 & 7.36 & 1908.02 & -12.84 & 1851.46 & -20.35 & 0.56 & 0.07 \\
\hline \multirow{2}{*}{ VIC } & Demand & 41.44 & 30.69 & 7478.68 & 3054.77 & 7542.93 & 3058.37 & 0.60 & 0.036 \\
& Price & 33.46 & 10.76 & 1837.24 & -48.72 & 1784.79 & -42.45 & 0.52 & 0.06 \\
\hline
\end{tabular}




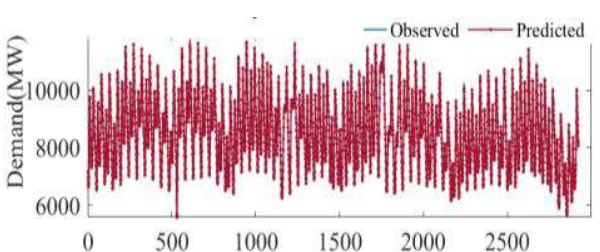

(a) NSW electricity demand

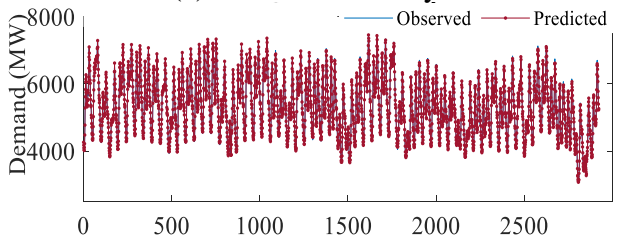

(c) VIC electricity demand

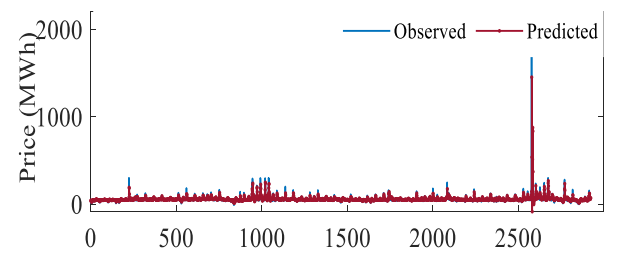

(b) NSW electricity price

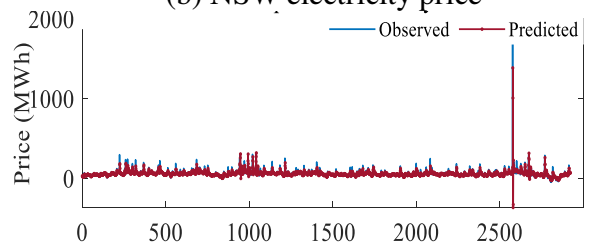

(d) VIC electricity price

Fig. 7. Electricity demand and price forecast based on the model output with the assumptions of the first scenario (a)-(b) NSW and second scenario (c)-(d) VIC.

To evaluate the reliability of the proposed LSTM model, the highest and lowest observed values from the test dataset are compared with the corresponding forecasted values. The forecasted highest and lowest electricity demand and price appear at the same time as the observed one in both scenarios. Both the highest records for demand and price for two scenarios were on a weekday and the lowest records were on weekends. The figures (relative error) in Table 1 show that in all forecast cases, the first scenario has a tendency to provide a better performance in demand values prediction and the second scenario is showing better result for price values. (Figs. 7 and 8).

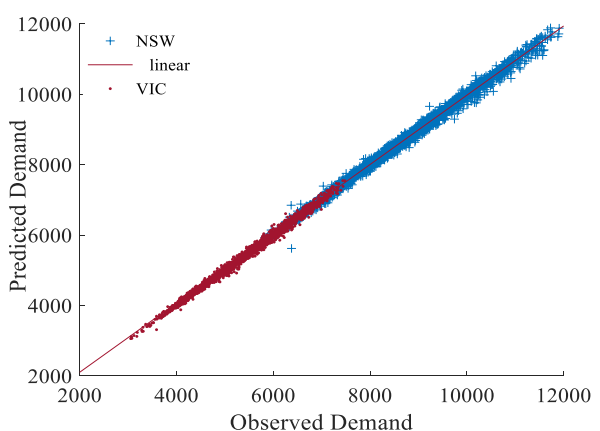

(a)

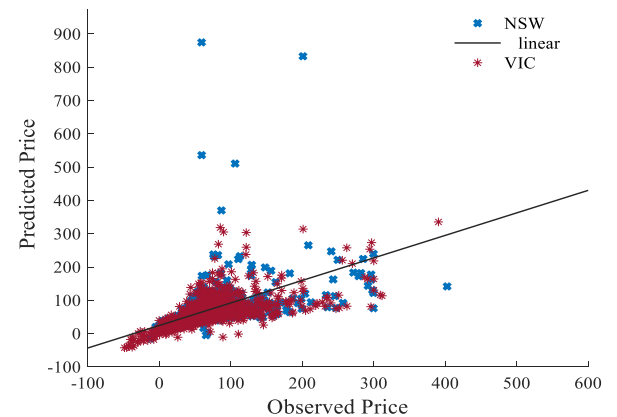

(b)

Fig. 8. Scatter plot to display coefficient correlation for electricity demand (a) and price (b) forecasting for both scenarios.

To validate the performance of the proposed LSTM network, two other widely used conventional forecast methods, Support Vector Machine (SVM) and Regression Tree (RT), are also performed using the same dataset. Table 2 outlines the best possible results for each model. The findings indicate that the proposed model achieves the best results and compared to SVM and RT, the RMSE index of the proposed model has been averagely improved by $37 \%$ and $60 \%$ respectively in the case of electricity demand; 
similarly, the MAE has been improved by $39 \%$ and $47 \%$ respectively. In the case of electricity price, the performance error RMSE shows an improvement of $19 \%$ and $22 \%$ compared with SVM and RT respectively; similarly, MAE has been improved by $20 \%$ and $32 \%$, respectively.

\begin{tabular}{ccccc|ccccc}
\multicolumn{10}{c}{ Table 2. Different methods of comparison } \\
\hline & Method & RMSE & MAE & $\mathrm{R}^{2}$ & & Method & RMSE & MAE & $\mathrm{R}^{2}$ \\
\hline NSW & SVM & 88.28 & 65.52 & .97 & VIC & SVM & 68.82 & 51.39 & .98 \\
Electricity & RT & 100.27 & 75.09 & .97 & Electricity & RT & 78.40 & 60.02 & .98 \\
Demand & LSTM & 58.21 & 40.51 & .99 & Demand & LSTM & 41.44 & 30.69 & .99 \\
\hline NSW & SVM & 32 & 9.78 & .49 & VIC & SVM & 48.23 & 12.76 & .55 \\
Electricity & RT & 41.96 & 12.47 & .12 & Electricity & RT & 39.56 & 13 & .53 \\
Price & LSTM & 29.67 & 7.36 & .78 & Price & LSTM & 33.46 & 10.76 & .88 \\
\hline
\end{tabular}

\section{Conclusion}

COVID-19 pandemic has significantly influenced people's lifestyle in many ways. First, in two scenarios, the implications of the pandemic are being analysed from comprehensive perspectives on electricity demand and price data. The results show that different restriction measures in both scenarios and their impact on people's activities have considerably changed the electricity demand and price profile distinctively. For example, NSW with the highest population and less restrictive measures has more demand and price reduction than VIC. Electricity demand has increased in July 2020 for both scenarios compared to the same period in 2019. Significant price reductions were observed on weekends by $48 \%$ for NSW and $47 \%$ for VIC due to lower demand, increased renewable output, and lower oil prices. During the pandemic, overall demand and price patterns have not changed significantly compared to the same period last year even the total electricity production has dropped beside the demand. Residential demand has increased due to shut-down, working from home setup, and winter weather; and commercial demand has reduced due to limited business activities. Since residential demand is related to weather than other industries and based on the Practice Theory [23], people's activities at resident would be expressed as routine recurrent trends in terms of electricity usage, regardless of irregularity. Therefore, secondly, this paper proposes a LSTM based sequence-to-sequence network model to forecast electricity demand and price considering the current uncertain pandemic situation. This model can manage variable input and output length, and effectively learns temporal correlation in the input sequence to model temporal structure simultaneously. A few traditional forecast models are comprehensively tested and compared to the proposed model on real market data. Simulation results prove the effectiveness of the proposed method over others with smaller errors and good accuracy despite its conceptual simplicity. Future work will be focused on the severity and long term effect of the COVID-19 pandemic on the electricity market since we are still experiencing the pandemic.

\section{References}

1. Adhikari, R. \& Agrawal, R.K.: An introductory study on time series modeling and forecasting. arXiv preprint arXiv:1302.6613 (2013). 
2. Sutskever, I., Vinyals, O. \& Le, Q.V.: Sequence to sequence learning with neural networks. Advances in neural information processing systems, 3104-3112 (2014).

3. LeCun, Y., Bengio, Y. \& Hinton, G.: Deep learning. Nature 521 (7553), 436-444 (2015).

4. Mikolov, T., Joulin, A., Chopra, S., Mathieu, M. \& Ranzato, M.A.: Learning longer memory in recurrent neural networks. arXiv preprint arXiv:1412.7753 (2014).

5. Graves, A., Mohamed, A.-r. \& Hinton, G.: Speech recognition with deep RNN networks. In: IEEE conference on acoustics, speech and signal processing, pp. 6645-6649 (2013).

6. Nelson, D.M., Pereira, A.C. \& de Oliveira, R.A.: Stock market's price movement prediction with LSTM neural networks. In: IEEE International joint conference on neural networks (IJCNN), pp. 1419-1426 (2017).

7. Le, X.-H., Ho, H.V., Lee, G. \& Jung, S.: Application of long short-term memory (LSTM) neural network for flood forecasting. Water 1(7), 1387 (2019).

8. Ugurlu, U., Oksuz, I. \& Tas, O.: Electricity price forecasting using recurrent neural networks. Energies 11 (5), 1255 (2018).

9. Lipton, Z.C., Berkowitz, J. \& Elkan, C.: A critical review of recurrent neural networks for sequence learning. arXiv preprint arXiv:1506.00019 (2015).

10. Hochreiter, S. \& Schmidhuber, J.: Long short-term memory. Neural computation 9 (8), 1735-1780 (1997).

11. Bouktif, S., Fiaz, A., Ouni, A. \& Serhani, M.A.: Optimal deep learning lstm model for electric load forecasting using feature selection and genetic algorithm: Comparison with machine learning approaches. Energies 11 (7), 1636 (2018).

12. Li, C., Ding, Z., Zhao, D., Yi, J. \& Zhang, G.: Building energy consumption prediction: An extreme deep learning approach. Energies 10 (10), 1525 (2017).

13. Tong, C., Li, J., Lang, C., Kong, F., Niu, J. \& Rodrigues, J.J.: An efficient deep model for day-ahead electricity load forecasting with stacked denoising auto-encoders. Journal of parallel and distributed computing (117) 267-273 (2018).

14. Marino, D.L., Amarasinghe, K. \& Manic, M.: Building energy load forecasting using deep neural networks. In: IECON 2016-42nd Annual Conference of the IEEE Industrial Electronics Society, pp. 7046-7051 (2016).

15. Sehovac, L. \& Grolinger, K.: Deep Learning for Load Forecasting: Sequence to Sequence Recurrent Neural Networks With Attention. IEEE Access 8, 36411-36426 (2020).

16. Gong, G., An, X., Mahato, N.K., Sun, S., Chen, S. \& Wen, Y.: Research on short-term load prediction based on Seq2seq model. Energies 12(16), 3199 (2019).

17. Kuo, P.-H. \& Huang, C.-J.: An electricity price forecasting model by hybrid structured deep neural networks. Sustainability 10 (4), 1280 (2018).

18. Srivastava, N., Hinton, G., Krizhevsky, A., Sutskever, I. \& Salakhutdinov, R.: Dropout: a simple way to prevent neural networks from overfitting. The journal of machine learning research 15 (1), 1929-1958(2014).

19. Aggregated Demand and Price Data, https://aemo.com.au/, last accessed 2020/09/28.

20. Hu, M.Y., Zhang, G., Jiang, C.X. \& Patuwo, B.E.: A cross-validation analysis of neural network out-of-sample performance in exchange rate forecasting. Decision Sciences 30 (1), 197-216 (1999).

21. Garreta, R. \& Moncecchi, G.: Learning scikit-learn: machine learning in python. Packt Publishing Ltd. (2013).

22. National Electricity Market, https://opennem.org.au/energy/nem/, last accessed 2020/09/26.

23. Stephen, B., Tang, X., Harvey, P. R., Galloway, S., \& Jennett, K. I.: Incorporating practice theory in sub-profile models for short term aggregated residential load forecasting. IEEE Trans. Smart Grid. 8(4), 1591-1598 (2015). 